Abstract P3-S1.36 Table 1 Concordance between tests used to diagnosis urethritis among men

\begin{tabular}{lcccr}
\hline & \multicolumn{2}{l}{ NAAT result } & & \\
\cline { 2 - 4 } Gram stain result & Gonorrhoea & Chlamydia & Negative & Total \\
\hline Gonorrhoea & 91 & 0 & 0 & 91 \\
NGU & $3^{*}$ & $87^{*}$ & 187 & $277^{*}$ \\
Negative & 14 & 26 & 195 & 235 \\
Total & $108^{*}$ & $113^{*}$ & 382 & 600 \\
\hline
\end{tabular}

*Three of these results were positive for both gonorrhoea and chlamydia.

Conclusion Gram stain is a reliable and relatively inexpensive test for the diagnosis of gonorrhoea among men. Men diagnosed with gonorrhoea or NGU using this point-of-care test were treated earlier than those diagnosed with NAAT only. The prevalence of atypical urethritis among this study population was high and further research should be conducted to investigate the possible aetiologies.

\section{P3-S1.37 THE ROLE OF MICROSCOPY IN THE DIAGNOSIS OF PROCTITIS AMONG MEN WHO HAVE SEX WITH MEN (MSM). A RETROSPECTIVE REVIEW OF RECTAL SCREENING}

doi:10.1136/sextrans-2011-050108.437

${ }^{1} \mathrm{P}$ Horner, ${ }^{2} \mathrm{D}$ Harte. ${ }^{1}$ University of Bristol, Bristol, UK; ${ }^{2}$ University Hospital Bristol trust, Bristol, UK

Background The rectum is a common site of infection with Chlamydia trachomatis (CT), Mycoplasma genitalium (MG) and Neisseria gonnorrhoeae (GC) among men who sex with men (MSM) who engage in receptive anal intercourse (RAI). Whilst the majority of individuals remain asymptomatic, these infections are likely to induce rectal inflammation which may have implications for HIV transmission. Information regarding the association between rectal CT, MG and GC and proctitis is limited.

Objectives To calculate the prevalence of proctitis among a cohort of MSM and investigate the association of proctitis with rectal CT and GC.

Methods Data were collected retrospectively. All MSM attending the clinic between July and December 2010 were included. Proctitis was defined as $>5$ PMNLs per high power field on microscopy of a Gram-stained rectal smear. Information including sexual history, clinical presentation, microscopy and GC/CT NAAT (Aptima combo 2 (AC2)) results were recorded on a proforma. Data were analysed using SPSS 16.0. Statistical tests of association were performed using Pearson's $\chi^{2}$ test. OR were calculated using logistic regression.

Results There were 425 MSM who attended for screening in the review period. Of these $83 \%$ were UK born. The median age was 32 years (range $=16-69 \mathrm{yrs}$ ). RAI in the preceding 3 months was reported by $59 \% \quad(n=251)$. Of these $64 \% \quad(n=160)$ had rectal microscopy performed. Rectal symptoms were reported by $11 \%$. The majority (96\%) of samples were collected using a blind rectal swab technique. Proctitis was diagnosed in 23 (14.4\%). Of these five were AC2-positive (CT =2, GC=1, CT +GC=1, LGV=1). Rectal AC2 was positive in $19 / 160(11.8 \%) \quad(\mathrm{GC}=7, \mathrm{CT}=10, \mathrm{CT}+\mathrm{GC}=1$, $\mathrm{LGV}=1$ ), five of whom had proctitis. Sensitivity and specificity of microscopy for rectal CT/ GC was $26 \%$ and $87 \%$ respectively. Factors which were predictive of proctitis included rectal symptoms $(p=0.001)$, and being HIV-1sero-positive $(p=0.001)$ but being CT/ GC/LGV positive was not associated ( $\mathrm{p}=0.157$ OR $2.4 ; 95 \% \mathrm{CI}=0.8$ to 7.5 ).

Conclusion Proctitis is common among MSM, particularly HIV-1 seropositive men. The association with GC/CT infection was weak.
The causes of and relevance of proctitis remain poorly understood and further research is warranted.

\section{P3-S1.38 MOLECULAR DETECTION OF CHLAMYDIA TRACHOMATIS AND NEISSERIA GONNORHOEA IN RECTAL SWABS AND (SELF) COLLECTED VAGINAL SWABS WITH THE COBAS 4800 SYSTEM}

doi:10.1136/sextrans-2011-050108.438

S Morre. VU University Meducal Center, Amsterdam, Netherlands

Objectives Chlamydia trachomatis (CT) and Neisseria gonnorhoea (NG) are the most prevalent sexual transmitted diseases worldwide. In recent years molecular diagnostics for the standard sample types like cervical swabs and urines has been highly adapted in most laboratories. Currently more difficult samples types, with regard to inhibition, sensitivity and specificity, like rectal swabs and (self collected) vaginal swabs are received more often in the laboratories. This study describes the performance of the new Cobas 4800 for the detection of CT and NG in these difficult sample types.

Methods A total of 1100 co-collected swabs were tested (900 vaginal; 200 rectal). Informed consent was obtained from all patients included in the study for co-collection. All swabs were tested routinely with the M2000 system (Abbott) and the cocollected samples were tested in the Cobas 4800 system (Roche). All swabs were collected in their corresponding transport buffer and tested blinded. Discrepant results were tested with an independent real time PCR method.

Results In the 900 vaginal swabs 10\% ( $n=90)$ were positive for CT and $0.5 \%(n=4)$ for NG and in the 200 rectal swabs $11 \%$ CT positives $(n=22)$ an $6 \%$ NG positives $(n=12)$ were identified. In the vaginal samples nine discrepant results between the Cobas 4800 and M2000 system were identified. Five with borderline values and four with clear different results (1 NG, 3CT). Two M2000 positive results (Ct 35.9; 32.3) were available for home brew analysis and could not be confirmed. In the rectal samples 8 discrepant results were found including three clear differences (2 NG; 1 CT). The CT discrepant sample was positive in the Cobas 4800 system (Cp 33.1) and negative in the M2000 system. For NG two samples were negative in the Cobas 4800 system but positive (Ct 23.8; 32.0) in the M2000 system. All three clear discrepant results were available for home brew analysis and confirmed the Cobas 4800 results.

Conclusion Both samples types showed a high concordance between the two systems (к 0.95 for CT; к 0.93 for NG). For detection of vaginal swabs some small differences were found both for CT and NG but these were equally spread between the two systems. There is no difference between self collected and clinician taken vaginal swabs. All clear rectal swabs discrepancies confirmed the Cobas 4800 results. This remarkable difference has to be further studied. In general, (self collected) vaginal swabs and rectal swabs show reliable results for routine detection of CT and NG.

\section{P3-S1.39 COMPARISON OF STRATEGIES FOR EMPIRIC CHLAMYDIA TRACHOMATIS (CT) TREATMENT IN THE DENVER METRO HEALTH CLINIC: TREAT MPC VS INCREASED WBC ON WET PREP}

doi:10.1136/sextrans-2011-050108.439

K Peterson, T Mickiewicz. Denver Public Health, Denver, USA

Background Our STD stat lab reports WBC on the vaginal wet prep as normal or increased ( $\geq 10 \mathrm{WBC} / \mathrm{LPF}$ ). WBC reflect inflammation and may be associated with CT infection. Mucopurulent cervicitis (MPC) may also result from CT, and is diagnosed by visualising the 
Abstract P3-S1.39 Table 1 Comparison of empiric treatment strategies using MPC or increased WBC on wet prep to direct treatment

\begin{tabular}{|c|c|c|c|c|c|c|c|}
\hline Strategy & $\begin{array}{l}\%(\mathrm{~N}) \mathrm{CT} \\
\text { cases } \\
\text { treated }\end{array}$ & $\begin{array}{l}\%(\mathrm{~N}) \mathrm{CT} \\
\text { cases } \\
\text { missed }\end{array}$ & $\begin{array}{l}\%(\mathrm{~N}) \text { missed } \\
\mathrm{CT} \text { cases other } \\
\text { strategy covers }\end{array}$ & $\begin{array}{l}\%(\mathrm{~N}) \text { treated } \\
\text { patients } \\
\text { without CT }\end{array}$ & $\begin{array}{l}\text { CT-negative } \\
\text { patients treated/CT } \\
\text { case treated }\end{array}$ & $\begin{array}{l}\text { CT-positive } \\
\text { cases missed/CT } \\
\text { case treated }\end{array}$ & $\begin{array}{l}\text { Time to treatment for missed } \\
\text { CT cases in } \% \text { Note: } 0 \text { days }=\text { treated } \\
\text { as contact day of visit }\end{array}$ \\
\hline Treat all MPC as CT & $13 \%(209)$ & $87 \%(1419)$ & $64 \%(907)$ & $77 \%(691)$ & 3.3 & 6.8 & $\begin{array}{l}0 \text { days: } 32 \% \\
1-14 \text { days: } 29 \% \\
15-30 \text { days: } 11 \% \\
31-90 \text { days: } 5 \% \\
\text { Not treated: } 23 \%\end{array}$ \\
\hline $\begin{array}{l}\text { Treat all increased } \\
\text { WBC as CT }\end{array}$ & $67 \%(1089)$ & $33 \%(539)$ & $5 \%(27)$ & $82 \%(4896)$ & 4.5 & 0.5 & - \\
\hline Treat all NGU as CT & $(1725)$ & - & - & $64 \%(3105)$ & 1.8 & - & - \\
\hline
\end{tabular}

cervix on speculum exam. We modelled what effect two strategies would have on empiric CT treatment in the Denver Metro Health Clinic (DMHC). Current strategy is to treat all MPC to cover possible CT. We compared that to expected results if all increased WBC on wet prep were treated to cover possible CT. Early treatment is thought to be important in preventing complications in women. The US Infertility Prevention Project guidelines call for treating 75\% of women by 14 days after testing, and $90 \%$ by 30 days.

Methods The DMHC is an urban STD clinic with an electronic medical record (EMR). The EMR was used to identify all women attending the DMHC and receiving a wet prep between 09/01/06 and $02 / 04 / 11$. They were divided into two groups by wet prep results (increased WBC vs normal WBC) and further divided by diagnoses of MPC and CT. Differences between the groups were assessed by $\chi^{2}$, and the sensitivity and specificity of increased WBC and MPC for CT were calculated. For each strategy, the \% of treated and missed cases of CT; \% of missed cases that would have been treated by the other strategy; \% of treated patients without CT; and the number of CT-negative patients treated, and CT-positive patients missed, for every CT case treated were calculated. NGU in men was used as a comparison where applicable. For CT cases that were missed by the MPC strategy, actual time to treatment was identified.

Results 19027 women were seen during this time and 12066 had a wet prep done. Of these, 5985 had increased WBC, 6081 had normal WBC, 900 had MPC and 1628 had CT. CT was positive in 1089 with increased WBC (182 had MPC) and 539 with normal WBC (27 had MPC). Both MPC and increased WBC were significantly associated with CT infection $(\mathrm{p}<0.0001)$ : CT positivity rates were $23.2 \%$ in women with MPC; $18.2 \%$ in women with increased WBC; and $8.7 \%$ in women with neither. The sensitivity and specificity of MPC for CT were $12.8 \%$ and $93.3 \%$ and for increased WBC 66.9\% and 53.1\%. See Abstract P3-S1.39 table 1 for comparison of the strategies.

Conclusions Increased WBC have poor specificity for CT but better sensitivity than MPC. Given greater delay in treatment with the MPC strategy, the increased WBC strategy is attractive for our clinic.

\section{P3-S1.40 FREQUENCY AND PREDICTORS OF RECOMMENDED GONORRHOEA THERAPIES IN WASHINGTON STATE}

doi:10.1136/sextrans-2011-050108.440

${ }^{1} \mathrm{R}$ Kerani, ${ }^{2} \mathrm{M}$ Stenger, ${ }^{2} \mathrm{M}$ Aubin, ${ }^{3} \mathrm{M}$ Golden. ${ }^{1}$ Public Health, Seattle, King County, USA; ${ }^{2}$ Department of Health, Washington, Olympia, USA; ${ }^{3}$ University of Washington, Seattle, USA

Background Washington State (WA) and CDC treatment guidelines recommend ceftriaxone (CFX) as the primary therapy for gonor- rhoea (GC), and some evidence suggests that azithromycin (AZM) plus an oral cephalosporin is superior to an oral cephalosporin alone in the treatment of GC.

Methods We used information from WA GC case report forms submitted July 2009-December 2010 to calculate the proportion of cases receiving different GC therapies. We used log regression to identify factors associated with recommended treatments.

Results Treatment data were available for 3910 (96\%) of 4066 reported GC cases; 2087 (53.4\%) were treated with CFX, 931 $(23.8 \%)$ with cefixime, (533) $13.6 \%$ with cefpodoxime, and 359 (9.2\%) with another drug. Of 1464 persons receiving an oral cephalosporin, 507 (34.6\%) also received AZM. Abstract P3-S1.40 table 1 displays the distribution of single and multidrug therapies for the three most common therapies observed. In a multivariate model, treatment with CFX was associated with male gender (prevalence ratio (PR): $1.23,95 \%$ CI 1.14 to 1.32 ), Asian/Pacific Islander race (PR: 1.10, $95 \%$ CI 1.00 to 1.20 ) and other/multiple races (PR: $1.13,95 \%$ CI 1.04 to 1.22) (vs White race). Compared to persons treated by private sector medical providers, CFX use was more common among persons treated in STD clinics (PR: 1.65, 95\% CI 1.53 to 1.77), ER/ urgent care clinics (PR: 1.37, 95\% CI 1.25 to 1.50), other hospital settings (PR: $1.27,95 \%$ CI 1.12 to 1.44 ), or community health centers (PR: 1.14, 95\% CI 1.00 to 1.29), and less common among those treated in family planning clinics (PR: $0.38,95 \%$ CI 0.30 to 0.47 ) and by other provider types (PR: $0.86,95 \%$ CI 0.76 to 0.98 ) Among persons treated with oral cephalosprins, concurrent treatment with AZM was associated with male gender (PR: $1.7195 \% \mathrm{CI}$ 1.45 to 2.01 ), treatment in an STD clinic (PR: $1.64,95 \%$ CI 1.41 to 1.91 ) or ER/urgent care clinic (PR: $1.28,95 \%$ CI 1.03 to 1.58 ) vs by a private provider, having GC only (PR:3.88, 95\% CI 2.84 to 5.28 ) (vs chlamydial coinfection), and Seattle residence (1.32, 95\% CI 1.08 to 1.61$)$

Abstract P3-S1.40 Table 1

\begin{tabular}{lrllll}
\hline & Total & $\begin{array}{l}\text { Single drug } \\
\text { therapy }\end{array}$ & With AZM & $\begin{array}{l}\text { With } \\
\text { doxyclycline }\end{array}$ & $\begin{array}{l}\text { With another } \\
\text { drug }\end{array}$ \\
\hline Ceftriaxone & 2087 & $773(37.0 \%)$ & $1051(50.4 \%)$ & $231(11.1 \%)$ & $32(1.5 \%)$ \\
Cefixime & 931 & $543(58.3 \%)$ & $334(35.9 \%)$ & $50(5.4 \%)$ & $4(0.3 \%)$ \\
Cefpodoxime & 533 & $334(62.6 \%)$ & $173(32.5 \%)$ & $25(4.7 \%)$ & $1(0.2 \%)$ \\
\hline
\end{tabular}

Conclusions Approximately half of all persons with gonorrhoea in WA do not receive CFX and over $20 \%$ receive an oral cephalosporin alone, which is not recommended in WA guidelines. Efforts to increase CFX should focus on identifying and surmounting barriers to the use of CFX, particularly in places such as family planning clinics and other settings where use is now low. 